\title{
Spotting rare items makes the brain "blink" harder: Evidence from pupillometry
}

\author{
Megan H. Papesh ${ }^{1}$ - Juan D. Guevara Pinto ${ }^{1}$ \\ Published online: 20 June 2019 \\ (C) The Psychonomic Society, Inc. 2019
}

\begin{abstract}
In many visual search tasks (e.g., cancer screening, airport baggage inspections), the most serious search targets occur infrequently. As an ironic side effect, when observers finally encounter important objects (e.g., a weapon in baggage), they often fail to notice them, a phenomenon known as the low-prevalence effect (LPE). Although many studies have investigated LPE search errors, we investigated the attentional consequences of successful rare target detection. Using an attentional blink paradigm, we manipulated how often observers encountered the first serial target (T1), then measured its effects on their ability to detect a following target (T2). Across two experiments, we show that the LPE is more than just an inflated miss rate: When observers successfully detected rare targets, they were less likely to spot subsequent targets. Using pupillometry to index locus-coeruleus (LC) mediated attentional engagement, Experiment 2 confirmed that an LC refractory period mediates the attentional blink ('Nieuwenhuis, Gilzenrat, Holmes, \& Cohen, 2005, Journal of Experimental Psychology: General, 134[3], 291-307), and that these effects emerge relatively quickly following T1 onset. Moreover, in both behavioral and pupil analyses, we found that detecting low-prevalence targets exacerbates the LC refractory period. Consequences for theories of the LPE are discussed.
\end{abstract}

Keywords Low-prevalence effect $\cdot$ Attentional blink $\cdot$ Locus coeruleus $\cdot$ Pupillometry

Abundant research has established that rare targets are harder to find than common targets are, a phenomenon known as the low-prevalence effect (LPE; 'Wolfe, Horowitz, \& Kenner, 2005; see 'Horowitz, 2017, for a meta-analysis). The LPE arises in laboratory (e.g., 'Wolfe et al., 2007) and applied contexts, such as radiology ('Evans, Birdwell, \& Wolfe, 2013), identification checks (e.g., 'Papesh, Heisick, \& Warner, 2018), and baggage screening ('Wolfe, Brunelli, Rubinstein, \& Horowitz, 2013). Because visual search is complex, involving overlapping and cascaded cognitive operations (e.g., oculomotor guidance, object identification, decision-making), a full theoretical account of the LPE must necessarily consider each stage.

The multi-decision model (MDM; 'Wolfe \& Van Wert, 2010) explains miss rates via two mechanisms - quitting thresholds and identification criteria. According to the MDM, observers make successive two-alternative forcedchoice decisions to fixated search items, querying whether

Megan H. Papesh

mpapesh@1su.edu

1 Department of Psychology, Louisiana State University, Baton Rouge, LA, USA each is a target. If the scrutinized item surpasses an identification criterion, the observer responds, "target present." If the item falls below criterion, observers repeat this process for a new item. As search continues, a simultaneous diffusion process accumulates toward a quitting threshold. Once this threshold is reached, observers terminate search. By this model, low-prevalence targets induce two changes: Quitting thresholds become relaxed (making early search termination more likely), and identification criteria become stricter (making it harder for observers to appreciate targets as "targets").

Ample evidence shows that the LPE causes observers to prematurely conclude search, consistent with reduced quitting thresholds. When targets appear infrequently, "target-absent" responses are faster (e.g., Godwin, Menneer, Riggs, Cave, Thaibsyah, \& Donnelly, 2015b; 'Rich et al., 2008; 'Wolfe et al., 2005), and eye-movement data indicate that rare targets often go unexamined (e.g., 'Peltier \& Becker, 2016; 'Wolfe \& Van Wert, 2010). Eye-movement studies also support prevalence-linked shifts in identification thresholds. For example, 'Hout, Walenchok, Goldinger, and Wolfe (2015) found that decision latencies were slower after observers fixated rare targets (see also Godwin, Menneer, Riggs, Cave, \& Donnelly, 2015a; 'Peltier \& Becker, 2016). This suggests that identification criteria increased, and observers required more evidence 
to produce "target-present" responses. 'Hout et al. also used rapid serial visual presentation (RSVP) to eliminate variation in participants' quitting thresholds and potential errors due to repetitive motor responses (e.g., 'Fleck \& Mitroff, 2007). Although participants responded after all items had been viewed, rare targets were still missed disproportionately often. Combined, these results reveal that both quitting thresholds and identification criteria contribute to the LPE. Although inflated miss rates are the hallmark of the LPE, our interest involved the consequences of successful rare target detection. Specifically, if identification criteria for rare targets are conservatively shifted, then surpassing those criteria should consume more cognitive resources, yielding consequences for other ongoing perceptual and cognitive processes.

Most LPE studies have examined visual search, measuring how target prevalence affects behavioral and oculomotor measures of target-present/target-absent decisions. For example, when targets co-occur, observers easily spot the common target yet often miss the rare one ('Fleck, Samei, \& Mitroff, 2010). Successfully detecting rare targets, however, should require extended (or exacerbated) attentional demands, selectively reducing available resources for subsequent cognitive processes. The present study investigated this hypothesis using the attentional blink (AB; 'Raymond, Shapiro, \& Arnell, 1992). The AB occurs when observers monitor a series of rapidly presented stimuli, searching for two targets. When the second target (T2) occurs in close temporal proximity (typically 200-500 ms) to the first target (T1), observers are likely to miss T2, due to ongoing resources devoted to T1 (see 'Martens \& Wyble, 2010).

Neurologically, the source of the $\mathrm{AB}$ has been attributed to the activity of the locus-coeruleus norepinephrine (LC-NE) system ('Nieuwenhuis et al., 2005; 'Warren et al., 2009). The LC is a brain-stem nucleus responsible for innervating nearly all forebrain regions with NE, with particularly strong connections to areas involved in attention ('Morrison \& Foote, 1986). Extensive axonal branching gives LC-NE neurons widespread influence, and volume transmission within diffusion zones ('Beaudet \& Descarries, 1978; 'Descarries, Watkins, \& Lapierre, 1977; 'O'Donnell, Zeppenfeld, McConnell, Pena, \& Nedergaard, 2012), coupled with different concentration-dependent adrenoreceptors ('Ramos \& Arnsten, 2007), allow the LC-NE system to modulate attentional states ('Aston-Jones \& Cohen, 2005; 'Aston-Jones, Rajkowski, \& Cohen, 1999, 2000; 'Gilzenrat, Holmes, Rajkowski, Aston-Jones, \& Cohen, 2002; 'Usher, Cohen, Servan-Schreiber, Rajkowski, \& Aston-Jones, 1999). Whereas some receptor types are excitatory under high NE concentrations, others are inhibitory at low/moderate levels ('Bickler \& Hansen, 1996; 'Egli et al., 2005). These excitatory and inhibitory effects essentially enhance the neural signal-tonoise ratio, such that most neural activity is quieted, allowing a subset of activated populations to take priority ('Foote,
Freedman, \& Oliver, 1975; 'Freedman, Hoffer, Woodward, \& Puro, 1977; 'Polack, Friedman, \& Golshani, 2013; 'Segal \& Bloom, 1976; 'Waterhouse \& Woodward, 1980). According to the neurocomputational LC-NE theory of the AB ('Nieuwenhuis et al., 2005), the "activated populations," are not location or stimulus-specific, but rather function to enhance attentional abilities in a temporally precise manner.

Although direct recording from human LC is not possible (see `Clewett, Huang, Velasco, Lee, \& Mather, 2018, for a recent account using functional imaging), single-cell studies with animals confirm a tight connection between LC activity and attention. For example, when motivationally relevant stimuli are processed, even within simple signal-detection tasks, LC neurons exhibit a period of intense, phasic activity ('Aston-Jones \& Bloom, 1981; 'Foote, Aston-Jones, \& Bloom 1980; 'Grant, Aston-Jones, \& Redmund, 1988; 'Joshi, Li, Kalwani, \& Gold, 2016; 'Rasmussen, Morilak, \& Jacobs, 1986; 'Sara \& Segal, 1991). These phasic bursts typically peak approximately $150 \mathrm{~ms}$ after target onset, and prior to the behavioral response ( Aston-Jones, Rajkowski, Kubiak, \& Alexinsky, 1994; 'Clayton, Rajkowski, Cohen, J\& AstonJones, 2004; 'Joshi et al., 2016). Importantly, approximately $50-100 \mathrm{~ms}$ following phasic NE release $(200-250 \mathrm{~ms}$ posttarget onset), the LC becomes autoinhibitory ('Aghajanian, Cedarbaum, \& Wang, 1977; 'Egan, Henderson, North, \& Williams, 1983; 'Washburn \& Moises, 1989), causing a refractory period during which stimulusdriven LC activity is rarely observed (Aston-Jones et al., 1994; 'Usher et al., 1999). Together, the temporal characteristics of the LC-NE system suggest a dynamic period of enhanced, and then suppressed, attentional abilities, as schematically outlined in Fig. 1.

As implied by Fig. 1, the pattern of LC activation, followed by autoinhibition, should have consequences for target

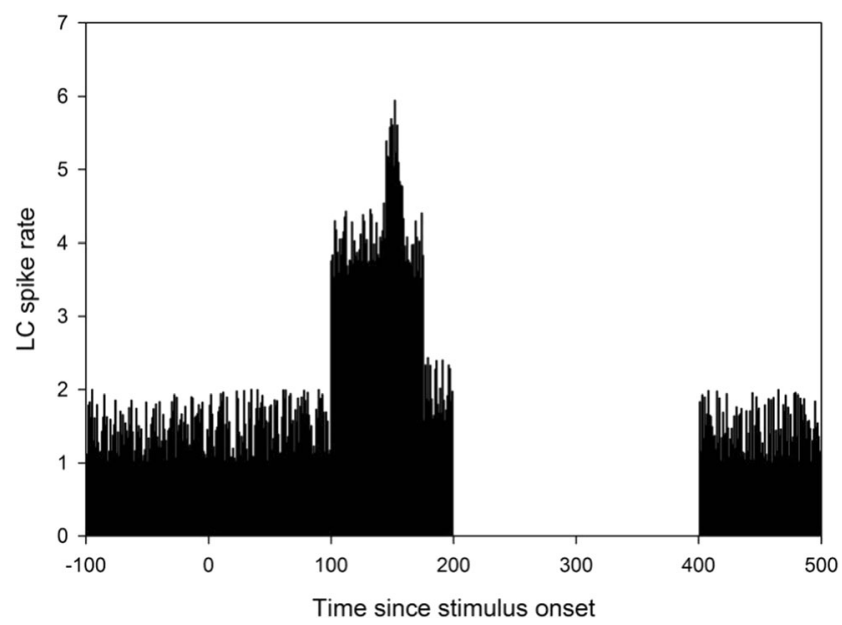

Fig. 1 Hypothetical locus-coeruleus (LC) spiking rate, time-locked to the onset of a motivationally salient stimulus. The LC refractory period typically lasts from approximately $200 \mathrm{~ms}$ poststimulus to $400-450 \mathrm{~ms}$ poststimulus ('Usher et al., 1999) 
detection in AB paradigms. Specifically, when T2 occurs within close ( $<150 \mathrm{~ms}$ ) temporal proximity to $\mathrm{T} 1$, that target should benefit from the residual LC activation and be easily detected (this is called Lag-1 sparing within the AB literature; see 'Visser, Biscof, \& Di Lollo, 1999, for a meta-analysis). Further, when T2 appears during the refractory period (between $200 \mathrm{~ms}$ and $400 \mathrm{~ms}$ post $\mathrm{T} 1$ ), no processing should occur, resulting in the AB. 'Nieuwenhuis et al. (2005) tested these predictions in a behavioral $\mathrm{AB}$ paradigm, confirming both Lag-1 sparing and the temporal dynamics of the $\mathrm{AB}$ (i.e., diminished detection at Lags 2 and 3, with a gradual recovery by Lag 5 ). Moreover, in an extension to the neurocomputational model of LC function proposed by 'Gilzenrat et al. (2002), 'Nieuwenhuis, Gilzenrat, Holmes, and Cohen (2005) showed that the model captured these critical characteristics, confirming a role for the $\mathrm{LC}$ in the $\mathrm{AB}$.

The neurocomputational model of $\mathrm{LC}$ function in the $\mathrm{AB}$ makes several additional predictions, as outlined by 'Nieuwenhuis et al. (2005). Critical to the present study, their simulations indicated that the magnitude of the $\mathrm{AB}$ should be influenced by the magnitude of the LC response, such that stimuli that yield larger LC responses should also yield a greater impairment to T2 detection. For example, stimulus novelty ('Sara \& Segal, 1991) and frequency (Aston-Jones, Rajkowski, \& Kubiak, 1997; 'Aston-Jones et al., 1994) have both been shown to modulate LC activity. It stands to reason, therefore, that these manipulations would also influence the magnitude of the $\mathrm{AB}$. In our study, we relied on the established temporal dynamics of the $\mathrm{AB}$ to provide insight into the resources consumed by rare and common targets, predicting that rare targets would demand enhanced LC activity and exacerbate the $\mathrm{AB}$.

Prior research investigating the influence of target probability on the $\mathrm{AB}$ has shown a prolonged $\mathrm{AB}$ with infrequent, relative to frequent, T1 (e.g., 'Crebolder, Jolicœur, \& Mcllwaine, 2002; 'Shapiro, Raymond, \& Arnell, 1994). In these studies, however, T1 was presented in a different color than the rest of RSVP search stream, so the effect of target probability may have been influenced by perceptual salience. Additionally, T2 was predictable (always $X$ or $Y$ ), so participants' decision was to determine whether an $X$ had been presented ('Shapiro et al., 1994), or to discriminate between two options ('Crebolder et al., 2002). In our study, we held perceptual salience constant and introduced greater ambiguity in the identity of $\mathrm{T} 2$, allowing us to more precisely investigate the impact of $\mathrm{T} 1$ probability on $\mathrm{T} 2$ processing. Across two experiments, we examined the attentional consequences of detecting rare, versus common, targets in a dual-target RSVP search paradigm. Our predictions derived from the LC-NE model of the AB ('Nieuwenhuis et al., 2005), such that we anticipated that low-prevalence $\mathrm{T} 1$ would demand greater $\mathrm{LC}$ resources and exacerbate the $\mathrm{AB}$, relative to high-prevalence $\mathrm{T} 1$. We tested these predictions behaviorally in Experiment 1, and with eye tracking in Experiment 2.

\section{Experiment 1}

Experiment 1 investigated the LPE in rapid serial visual search. Whereas many studies explore the LPE as it manifests in miss rates, we examined the consequences of successful rare target detection. Specifically, we derived hypotheses from the LC-NE model of the AB ('Nieuwenhuis et al., 2005), which suggests that stimuli that demand greater LC engagement lead to a prolonged LC refractory period. This refractory period typically lasts up to $400 \mathrm{~ms}$ ('Usher et al., 1999), which encompasses the "blink" duration in standard AB paradigms ('Martens \& Wyble, 2010). We hypothesized that, relative to common $\mathrm{T} 1$, rare $\mathrm{T} 1$ would demand greater LC engagement, and thus exacerbate the $\mathrm{AB}$. If prevalence exerts this effect by extending the refractory period, then we should observe an interaction between prevalence and lag. If, on the other hand, prevalence effects consume more attentional resources, without extending the refractory period, then we should observe a main effect of prevalence.

\section{Method}

Participants A power analysis (main effects and interaction $\alpha$ $=.05, \beta-1=.95$ ) conducted on the Prevalence $\times$ Target Presence interaction effect size in Experiment 2 of 'Peltier and Becker $\left(2016 ; \eta_{\mathrm{p}}{ }^{2}=.589\right)$ indicated that 25 participants were necessary to obtain a within-subjects LPE. Thus, 63 undergraduate students ( $M_{\text {age }}=19.48$ years, $S D=3.21 ; 44$ women $)$ participated in Experiment 1 for partial course credit. All participants self-reported normal or corrected-to-normal vision and color vision.

Stimuli Targets consisted of uppercase letters (excluding I, L, $\mathrm{O}, \mathrm{W}, \mathrm{X})$, whereas distractors consisted of digits (2-9) and symbols $(<,>,=, \%, \&, *, \#$, ?). To manipulate $\mathrm{T} 1$ prevalence, a subset of five letters (A, E, M, R, Y) appeared with unequal frequency. The letter $M$ was the high-prevalence target, appearing in 120 trials (50\%), the letters $E$ and $Y$ were medium-prevalence targets, each appearing in 48 trials (40\% total), and the letters $A$ and $R$ were low-prevalence targets, appearing in 12 trials each $\left(10 \%\right.$ total). ${ }^{1}$ The identity of T2 was completely random using the remaining letters of the alphabet, with each letter used equally across trials. All stimuli were presented in black, 32-point Courier New font on 21.5inch monitors, with 1,920 × 1,080 screen resolution and 60$\mathrm{Hz}$ sampling rates. Experimental procedures were controlled using E-Prime 2.0 ('Psychology Software Tools, 2012).

\footnotetext{
${ }^{1}$ Throughout, we use prevalence to refer to the presentation frequencies for different targets. Although frequency is the more appropriate term, we wanted to avoid confusion with lexical stimuli (i.e., high-frequency and low-frequency words) and remain consistent with the literature on visual search.
} 
Procedure After providing written informed consent and completing seven practice trials, participants completed a single block of 240 experimental trials. Participants self-initiated each trial by pressing the space bar, at which point a $500-\mathrm{ms}$ fixation cross appeared in the center of the screen. The fixation cross offset was followed by a 24-item RSVP stream, with each item presented centrally for $15 \mathrm{~ms}$, followed by a blank interstimulus interval (ISI) of $75 \mathrm{~ms}$, resulting in a presentation rate of 11.11 items/s (consistent with timing parameters in other studies; see 'Raymond et al., 1992). RSVP streams consisted of 22 distractor items and two targets. T1 only appeared in one of seven serial positions $(3,5,9,11,13,15,19)$, randomly selected per trial, ensuring that no target was presented in the first or last two positions. The lag between T1 and $\mathrm{T} 2$ was manipulated, such that six randomly selected lags $(2,3,4,5,6,7)$ occurred equally often. ${ }^{2}$ As is typical in $\mathrm{AB}$ research ("Martens \& Wyble, 2010), "lag" refers to the serial position at which T2 occurs after T1, so that "Lag 2" means that there was one intervening item between $\mathrm{T} 1$ and $\mathrm{T} 2$. Long lags (four and above) never occurred when $\mathrm{T} 1$ was presented at Serial Position 19. Following the offset of the RSVP stream, participants identified the two letters that appeared in the stream by typing them on the keyboard. Participants could enter letters in any order (T1 first or T2 first), and were asked to type " $\mathrm{X}$ " if they missed one or both letters. No feedback on their identification responses was provided.

\section{Results}

Accuracy was calculated based on correct reporting of target identities, regardless of the order in which participants typed their responses. Because accuracy data were proportions, they were arcsine-square-root transformed prior to analysis. For the purpose of clarity, we report inferential statistics on the transformed data, but present raw values in our descriptive statistics and graphs. The alpha level for all analyses was .05, multiple comparisons were subject to Bonferroni corrections, and degrees of freedom were Greenhouse-Geisser corrected, where necessary. Traditional frequentist analyses were supplemented with Bayesian versions conducted in JASP ('JASP Team, 2018). For Bayesian ANOVAs, the Bayes factor $\left(\mathrm{BF}_{10}\right)$ reflects the strength of evidence favoring the inclusion of each effect or interaction in a comparison model relative to a "null" model. Lastly, because identification accuracy did not differ significantly across the two target letters in the low-prevalence, $t(62)=1.62, p=.11$, or medium-prevalence, $t(62)=.66, p>.250$, conditions, we analyzed data for the two letters together, within each prevalence condition.

\footnotetext{
${ }^{2}$ Note that, due to a programming error, Lag 1 was not included, so we could not test predictions about Lag-1 sparing in Experiment 1.
}

T1 identification We analyzed T1 accuracy in a 3 (prevalence: high, medium, low) $\times 6$ (lag: $2,3,4,5,6,7$ ) repeatedmeasures ANOVA. Consistent with the LPE in visual search, we observed a reliable main effect of prevalence, $F(1.68$, 97.34) $=28.66, p<.001, \eta_{\mathrm{p}}^{2}=.33, \mathrm{BF}_{10}=2.094 \mathrm{e}+15$. Although the difference between raw low-prevalence (LP) and medium-prevalence (MP) T1 identification rates was small (see Fig. 2, left panel), post hoc tests revealed that this was a reliable difference, $t=-5.51, p<.001, \mathrm{BF}_{10}=172.42$. Importantly, participants' ability to identify high-prevalence (HP) T1 was reliably better than both LP, $t=2.84, p=.01$, $\mathrm{BF}_{10}=45.66$, and MP, $t=13.16, p<.001, \mathrm{BF}_{10}=3.789 \mathrm{e}+31$, T1. We also observed a small effect of lag, $F(4.32,250.36)=$ 2.56, $p=.04, \eta_{\mathrm{p}}^{2}=.04, \mathrm{BF}_{10}=0.04$, which revealed that participants' were less likely to identify $\mathrm{T} 1$ when $\mathrm{T} 2$ occurred at Lag 2, although this was only reliable when contrasted with Lag 5, $t=-3.32, p=.02, \mathrm{BF}_{10}=1.35$. This finding is consistent with prior research (e.g., 'Potter, Staub, \& O'Connor, 2002; 'Warren et al., 2009) suggesting that when T1 and T2 occur in close temporal proximity, the competition for limited attentional resources is resolved by devoting those resources to T2. There was no statistically reliable interaction, $F(6.67$, $413.33)=1.44 p=.19, \eta_{\mathrm{p}}^{2}=.02, \mathrm{BF}_{10}=0.01$.

T2 identification To examine whether successful identification of rare targets demands more resources than frequent targets, we conducted a 3 (T1 prevalence: HP, MP, LP) $\times 6(\mathrm{lag})$ repeated-measures ANOVA on the proportion of correct T2 identifications, contingent upon successful $\mathrm{T} 1$ identification (T2|T1). Results revealed main effects of T1 prevalence, $F(1.42,88.11)=11.5, p<.001, \eta^{2}=.15, \mathrm{BF}_{10}=99.61$, and lag, $F(5,310)=26.1, p<.001, \eta^{2}=.29, \mathrm{BF}_{10}=2.031 \mathrm{e}+27$, with no interaction, $F(5.63,349.2)=1.91, p=.08, \eta^{2}=.03$, $\mathrm{BF}_{10}=0.04$. Bonferroni-corrected pair-wise comparisons revealed that $\mathrm{T} 2$ identification did not significantly differ following detection of LP $(M=.70, S E=.02)$ or MP T1 $(M=$ $.72, S E=.01)$ when collapsed across all lags, $t=.74, p=1.00$, $\mathrm{BF}_{10}=0.05 .{ }^{3}$ However, relative to HP T1 $(M=.79, S E=.01)$, both LP, $t=6.73, p<.001, \mathrm{BF}_{10}=5.518 \mathrm{e}+7$, and MP T1, $t=$ 4.02, $p<.001, \mathrm{BF}_{10}=88.62$, produced lower $\mathrm{T} 2$ identification. The main effect of lag was characterized by a general improvement in performance with longer lags.

\section{Discussion}

Experiment 1 confirmed the prediction that the frequency with which $\mathrm{T} 1$ is encountered will affect the magnitude of the $\mathrm{AB}$. Specifically, the LC-NE model ('Nieuwenhuis et al., 2005) suggests that the $\mathrm{AB}$ will be modulated by any stimulus or

\footnotetext{
${ }^{3}$ Note that the difference in T2 identification between LP and MP T1 is reliable when examining raw accuracy values, $t=2.62, p=.03$, such that $\mathrm{T} 2$ identification decreased following the detection of LP, relative to MP, T1.
} 


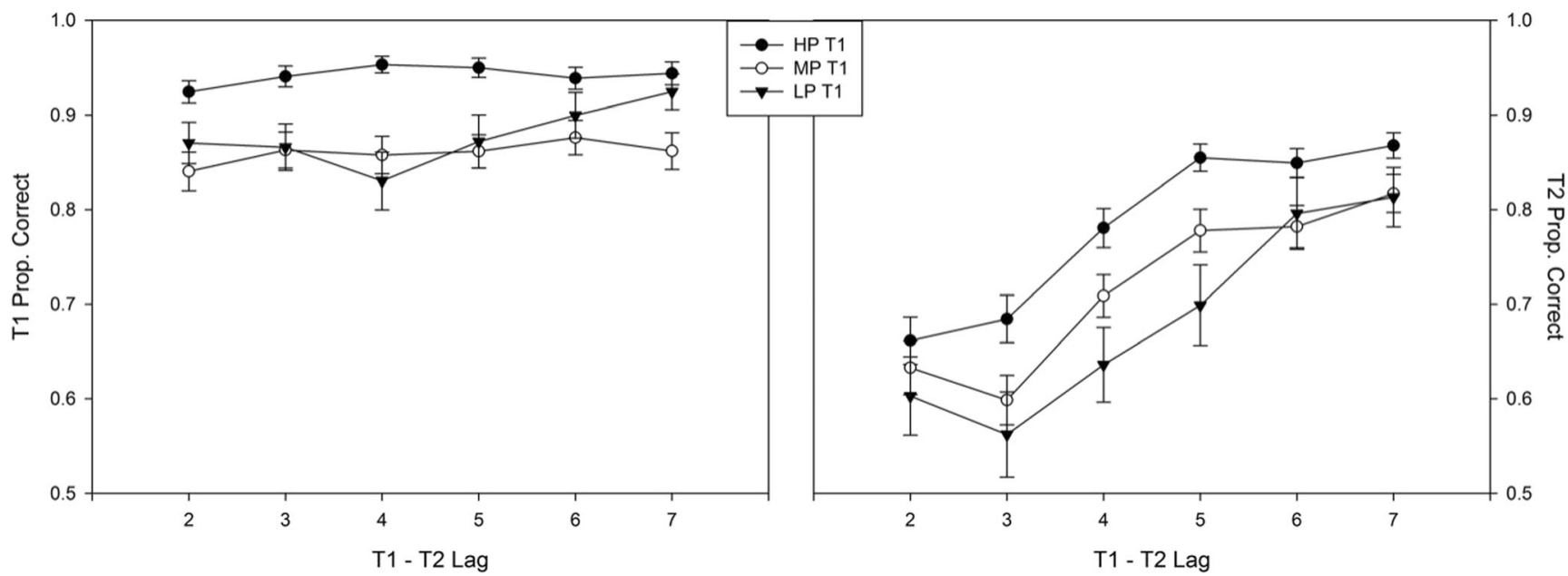

Fig. 2 Results for the proportion of correct T1 identifications (left panel) and correct T2 identifications on trials were T1 was also correctly identified (T2|T1; right panel) in Experiment 1. Error bars represent $\pm 1 S E M$

variable that differentially engages the LC, such as stimulus frequency. In Experiment 1, we showed that infrequent $\mathrm{T} 1$ were less likely to be detected than frequent $\mathrm{T} 1$, and that successful rare target detection induced a greater attentional impairment, as reflected by observers' greater inability to detect a second target after the first. Although the interaction between Prevalence and Lag was not reliable, and therefore cannot conclusively support the prediction that rare targets extend the LC refractory period, the lag-3 data in Figure 2 (right panel), hints toward this effect. The influence of target rarity on the $\mathrm{AB}$ is not without precedent ('Crebolder et al., 2002; 'Shapiro et al., 1994), and our results generally replicate prior findings, using equally perceptually salient $\mathrm{T} 1$, and greater ambiguity in the identity of $\mathrm{T} 2$.

Although we relate our findings from Experiment 1 to the neurocomputational model of the LC, direct recordings from the LC are nearly impossible in humans. Whereas pharmacological and microelectrode research are common for testing LC functions in non-human animals, the dominant method for estimating LC activity in humans derives from the close relationship between the LC and pupillary responses. When researchers can minimize external influences (e.g., lighting, variable temperatures) and internal factors (e.g., fatigue, pharmacological influence, emotional arousal), the size of the eyes' pupils can be traced to activity in the LC-NE system, with greater pupil dilation reflecting greater LC activation (Gilzenrat, Nieuwenhuis, Jepma, \& Cohen, 2010). For example, direct neural recordings from awake, behaving animals often link pupil size to the activity of the LC-NE system (e.g., 'Aston-Jones \& Cohen, 2005). 'Joshi et al. (2016) recently reported that LC activity reliably precedes both spontaneous and stimulus-driven pupillary changes. Moreover, these changes occurred on a time-scale comparable to single-unit spiking rates, with pupil dilation occurring approximately $310 \mathrm{~ms}$ after a spike and constriction occurring
$750 \mathrm{~ms}$ post-spike. During passive fixation, pupil dilation was directly correlated with LC spike rates. When a startling tone was played, LC activity transiently increased, directly before pupil dilation.

Previous research has clearly linked LC activity to the AB, using both behavioral ('Warren et al., 2009) and computational modeling approaches ('Nieuwenhuis et al., 2005). Subsequent work has confirmed this link, and elucidated the relatively rapid pupillary responses to RSVP stimuli ('Wierda, Van Rijn, Taatgen, \& Martens, 2012; 'Zylberberg, Oliva, \& Sigman, 2012). In Experiment 2, we used pupillometry to more precisely test the predictions derived from the LC-NE hypothesis about the $\mathrm{AB}$.

\section{Experiment 2}

The goal of Experiment 2 was to test predictions from the LCNE hypothesis about the impact of target prevalence on the AB. The LC typically exhibits a pattern of phasic activity that peaks within $150 \mathrm{~ms}$ of the onset of motivationally-relevant stimuli ('Aston-Jones et al., 1994; 'Clayton et al., 2004; 'Joshi et al., 2016). Approximately $200-250 \mathrm{~ms}$ following stimulus onset, the LC becomes refractory, such that that new stimuli rarely evoke LC activity ('Aston-Jones et al., 1994; 'Usher et al., 1999). This time course closely matches that of the AB. Specifically, if a second target is presented during the phasic LC response to a first target (within $100-150 \mathrm{~ms}$; 'Aston-Jones et al., 1994; 'Clayton et al., 2004), T2 processing should be facilitated (lag-1 sparing) ${ }^{4}$. If, however, T2 is presented during the LC refractory period $(200-400 \mathrm{~ms}$ after T1), it should go undetected (attentional blink).

\footnotetext{
${ }^{4}$ Note that this prediction is not unique to the LC-NE hypothesis.
} 
According to the LC-NE hypothesis, LC activity drives both lag-1sparing and the $\mathrm{AB}$. As such, factors that differentially engage the LC should produce observable changes in these phenomena. Because the LC is sensitive to stimulus frequency ('Aston-Jones et al., 1997; Aston-Jones et al., 1994), we hypothesized that detecting rare T1 should increase lag-1 sparing and strengthen the LC refractory period, thereby exacerbating the attentional blink. Given the temporal dynamics of LC activity, rare $\mathrm{T} 1$ should induce worse $\mathrm{T} 2$ detection between 200 and $400 \mathrm{~ms}$ of $\mathrm{T} 1$ onset. To approximate LC engagement, we monitored pupil size in Experiment 2, because it is an established and time-sensitive marker of LC activity ('Joshi et al., 2016), and should therefore also be sensitive to stimulus frequency. Specifically, we hypothesized that pupillary responses should be larger for rare, relative to common, $\mathrm{T} 1$ detection, particularly in trials in which $\mathrm{T} 2$ is subsequently missed (AB trials). Given the approximate $300-\mathrm{ms}$ lag between LC activity and phasic pupillary responses, these effects should begin to emerge between $500-$ $700 \mathrm{~ms}$ post-T1 onset.

\section{Method}

Participants Fifty-one undergraduate students $\left(M_{\mathrm{age}}=20.7\right.$ years, $S D=4.75 ; 33$ women) participated in exchanged for partial course credit. A power analysis (within-subject factors $\alpha=.05, \beta-1=.95)$ indicated that "medium" size effects (Cohen's $f=0.257$ ) should be observed with the current sample size. All participants self-reported normal or corrected-tonormal vision and color vision.

Stimuli and apparatus Stimuli were presented in black (RGB: 0,0,0) 62-point New Courier font (approx. $3.5 \mathrm{~cm}$ ) on a silver background (RGB: 192, 192, 192) of a 27 -inch BenQ XL2720Z monitor with a resolution of $1920 \times 1080$ and a refresh rate of $60 \mathrm{~Hz}$. This was constant across all levels of target-prevalence. However, because we observed no differences between LP and MP T2|T1 identification rates in Experiment 1 , only low-prevalence (22\%; letters $A$ and $R$ ) and high-prevalence targets $(88 \%$; letter $M$ ) were included in this Experiment. Pupil sizes were recorded monocularly from each observers' dominant eye with an EyeLink 1000Plus eyetracker (SR Research Ltd., Mississauga, Ontario, Canada), with the sampling rate set to $500 \mathrm{~Hz}$. Viewing distance was $94 \mathrm{~cm}$ from the display and experimental procedures were controlled using E-Prime 2.0 ('Psychology Software Tools, 2012).

Procedure The procedure was similar to Experiment 1, except that $\mathrm{T} 2$ could now appear at Lag 1 (i.e., immediately following $\mathrm{T} 1$ ), late-appearing $\mathrm{T} 1$ could be followed by long-lag T2, and the search stream was lengthened to 28 items to accommodate the temporal dynamics of pupillary responses. T1 was presented at random in serial positions $6-14$, and $\mathrm{T} 2$ was presented at random Lags of 1-6, with an equal number of presentations across all six lags. The experiment consisted of four blocks of 54 trials (216 trials total), with self-paced breaks in between each block to ensure participant comfort on the eyetracker. Using blocks allowed us to ensure that $\mathrm{T} 1$ prevalence was consistently manipulated (42 HP trials, and 12 LP trials), such that random sampling did not produce phases with higher or lower prevalence. All other experimental procedures were identical to Experiment 1.

\section{Results}

T1 identification We examined T1 accuracy in a 2 (Prevalence) x $6(\mathrm{Lag})$ repeated measures ANOVA. We did not observe a main effect of Prevalence $\left(p>.250, \mathrm{BF}_{10}=\right.$ $0.091)$, but we observed a main effect of Lag, $F(4.15$, $205.77)=16.61, p<.001, \eta_{\mathrm{p}}^{2}=.25, \mathrm{BF}_{10}=9.146 \mathrm{e}+9$. Replicating the Lag effect from Experiment 1, we found that $\mathrm{T} 1$ was less likely to be identified when $\mathrm{T} 2$ occurred in close temporal proximity (Lag 1), relative to other Lags (see Figure 3, left panel). There was no interaction between Prevalence and Lag, $F(2,259)=1.58, p=.17, \eta_{\mathrm{p}}{ }^{2}=.03$, $\mathrm{BF}_{10}=0.072$.

T2 identification Although there was no reliable behavioral difference between HP and LP T1 identification, we predicted that participants' $\mathrm{T} 2$ identification would be reliably affected by the frequency with which successfully identified $\mathrm{T} 1$ were encountered. To test this hypothesis, we examined T2|T1 identification accuracy in a 2 (T1 Prevalence) x 6 (Lag) repeated measures ANOVA. Both main effects and the interaction were reliable. The main effect of Lag, $F(3.64,181.9)=$ $57.1, p<.001, \eta_{\mathrm{p}}^{2}=.53, \mathrm{BF}_{10}=9.869 \mathrm{e}+57$, manifest as lag-1 sparing (all pairwise comparisons reliable at $p<.001$ ), and then a gradual recovery in performance following the biggest detriment at Lag 3. The main effect of T1 Prevalence, $F(1,50)$ $=14.89, p<.001, \eta_{\mathrm{p}}^{2}=.23, \mathrm{BF}_{10}=10.52$, must be interpreted within the context of the small, but reliable interaction, $F(4.65$, 232.47) $=4.04, p=.002, \eta_{\mathrm{p}}{ }^{2}=.08, \mathrm{BF}_{10}=0.34$. As shown in the right panel of Figure 3, LP T1 did not produce more lag-1 sparing, $t=0.87, p>.250, \mathrm{BF}_{10}=0.087$, but induced a bigger detriment to T2 identification than HP T1 across nearly all lags. Pairwise comparisons revealed that the prevalence effects were reliable at $\mathrm{Lag} 2, t=2.63, p=.011, \mathrm{BF}_{10}=6.69$, $\operatorname{Lag} 3, t=4.53, p<.001, \mathrm{BF}_{10}=1133.39$, and $\operatorname{Lag} 4, t=2.24, p$ $=.03, \mathrm{BF}_{10}=2.90$, but not Lag $5, t=1.79, p=.08, \mathrm{BF}_{10}=$ 1.278 , or Lag $6, t=1.29, p=.202, \mathrm{BF}_{10}=0.595$.

\footnotetext{
${ }^{0}$ We also used the subtractive baseline correction method on normalized ( $z$ score) pupil sizes, yielding nearly identical waveforms (see the inset graph in Fig. 4).
} 


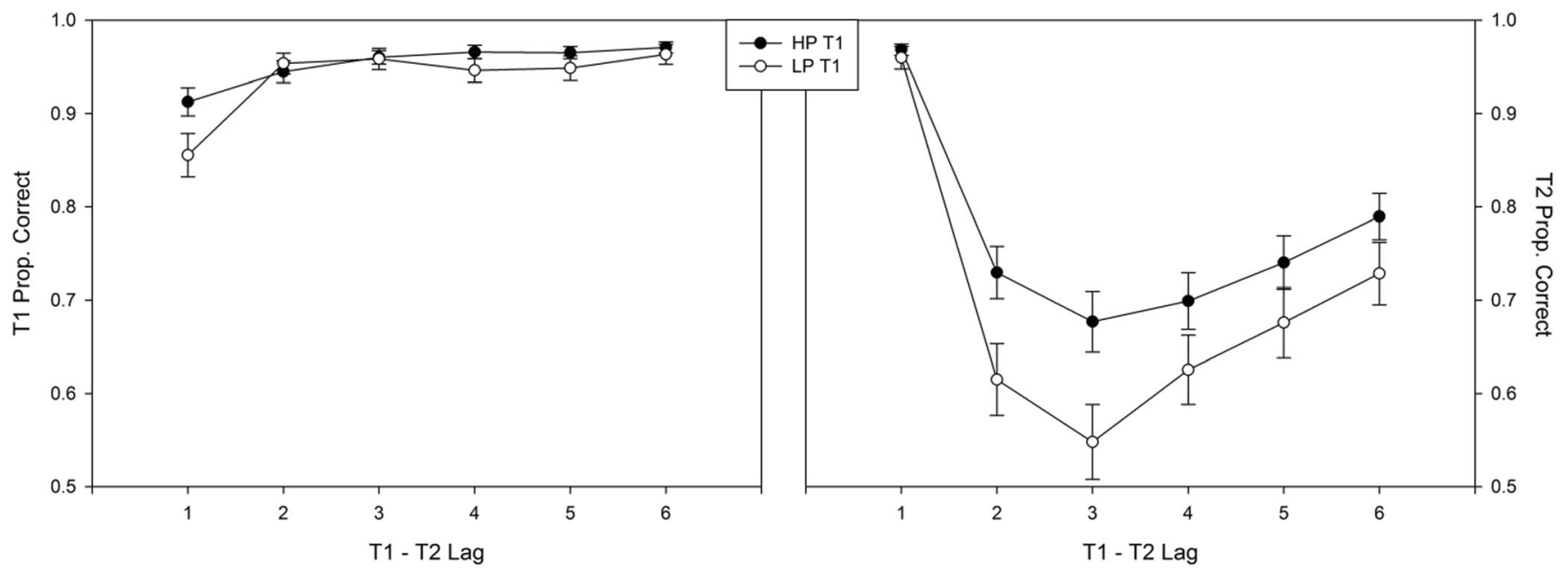

Fig. 3 Results for the proportion of correct T1 identifications across blocks (left panel) and correct T2|T1 identifications (right panel) in Experiment 2. Error bars represent $\pm 1 S E M$

Pupillometry analyses Pupil data were measured in arbitrary units standard to EyeLink trackers, and preprocessed and analyzed in MATLAB, using CHAP ('Hershman, Henik, \& Cohen, 2019). Preprocessing involved excluding outlier samples and trials using CHAP defaults (samples more than 2.5 $\mathrm{SD}$ from the trial-normalized mean and trials containing more than $20 \%$ outlier samples). Artifacts due to blinks were corrected by linear interpolation. Prior to preprocessing, we established 5 as the minimum acceptable number of valid trials per condition, which resulted in the exclusion of 7 participants. Another 2 participants were excluded because of errors in event messaging. From the remaining 42 participants, analyses retained $93 \%$ of HP trials and $91 \%$ of LP trials, with no participant contributing fewer than $70 \%$ of the total trials. Samples were baseline corrected by subtracting the average dilation during the trial period occurring 250-ms prior to the onset of T1, yielding relative pupil changes throughout the interest period ${ }^{5}$. As shown in Figure 4, trials were aligned to $\mathrm{T} 1$ onset (time 0 ), and our interest period ended at the offset of the final RSVP item.

To examine the hypothesis that detecting infrequent targets produce a "stronger" LC response, and therefore an exacerbated attentional blink, we first examined relative mean pupil sizes as a function of both Prevalence and whether participants' behavior reflected the typical AB pattern (i.e., whether they missed T2). These values were analyzed in a 2 (T1 Prevalence) x 2 (Blink: AB/no AB) repeated measures ANOVA. Although we predicted an interaction between Prevalence and Blink, such that LP targets should have yielded the largest pupils in AB trials, this interaction was not observed, $F(1,41)=0.79$, $p=.38, \eta_{\mathrm{p}}^{2}=.02, \mathrm{BF}_{10}=0.09$. As shown in Figure 5, there were no main effects of Blink, $F(1,41)=.45, p=$ $.51, \eta_{\mathrm{p}}^{2}=.01, \mathrm{BF}_{10}=0.23$, or Prevalence, $F(1,41)=$ $1.77, p=.19, \eta_{\mathrm{p}}^{2}=.04, \mathrm{BF}_{10}=0.38$.
As shown in Figure 4, the pupillary response to cognitive events occurs over an extended time course, so our trial-level analyses may have masked transient effects. To better explore the dynamics of the LPE and AB, we examined time-series analyses in CHAP. These analyses provide Bayesian (Bayes Factors) statistics for paired samples $t$-tests comparing two conditions as a function of trial time (Hershman et al., 2019). Whereas we observed no reliable Prevalence effect in the omnibus analysis (Figure 5), temporal analyses revealed that Prevalence effects emerged late in the stream, immediately prior to the onset of the response prompt (see Figure 6). Figure 6 also reveals the time-course over which the AB is revealed by pupil size. Whereas the earliest "peak" in the Bayes Factors may reflect phasic activity in response to target onset, the $\mathrm{AB}$ was revealed by enlarged pupils emerging (and dissipating) between $700-900 \mathrm{~ms}$ after T1.

Although Figure 6 shows evidence in favor of predicted main effects, our overarching hypothesis was that T1 prevalence would interact with the $\mathrm{AB}$ to produce the most enlarged pupils in LP trials. To evaluate this, and related, hypotheses, we plotted $\mathrm{BF}_{10}$ values for three additional hypotheses (see Figure 7): (1) In blink trials, LP T1 should yield larger pupils than HP T1, (2) within LP trials, those with an AB should be associated with larger pupils than those without an $\mathrm{AB}$, and (3) within HP trials, those with an AB should be associated with larger pupils than those without an AB. As shown in Figure 7, the evidence was mixed. We obtained moderate support for the first hypothesis, consistent with the temporal analyses shown in Figure 6. Specifically, the $\mathrm{BF}_{10}$ values supported the hypothesis that LP AB trials would yield larger pupils than HP AB trials, but only very late in the stream, possibly reflecting differential preparatory processes in anticipation of the response prompt. There was little evidence in favor of the hypothesis that HP AB trials would yield larger pupils than HP trials with no blink (hypothesis 3). Importantly, 




Fig. 4 Average baseline-corrected pupil size in arbitrary and normalized (inset graph) units. Time 0 denotes T1 onset. Separate lines show pupil size during high-prevalence (HP) and low-prevalence (LP) T1 trials that induced an attentional blink (AB) or not (no AB)

however, we obtained strong evidence in favor of the second hypothesis, that LP AB trials would yield larger pupils than LP trials with no blink. During LP blink trials, participants' pupils were markedly larger starting at $500 \mathrm{~ms}$ post-T1. Considered in conjunction with the time-series analysis in Figure 6, this suggests that the $\mathrm{AB}$ effects observed in the broader data are more reflective of LP blink trials, relative to HP blink trials.

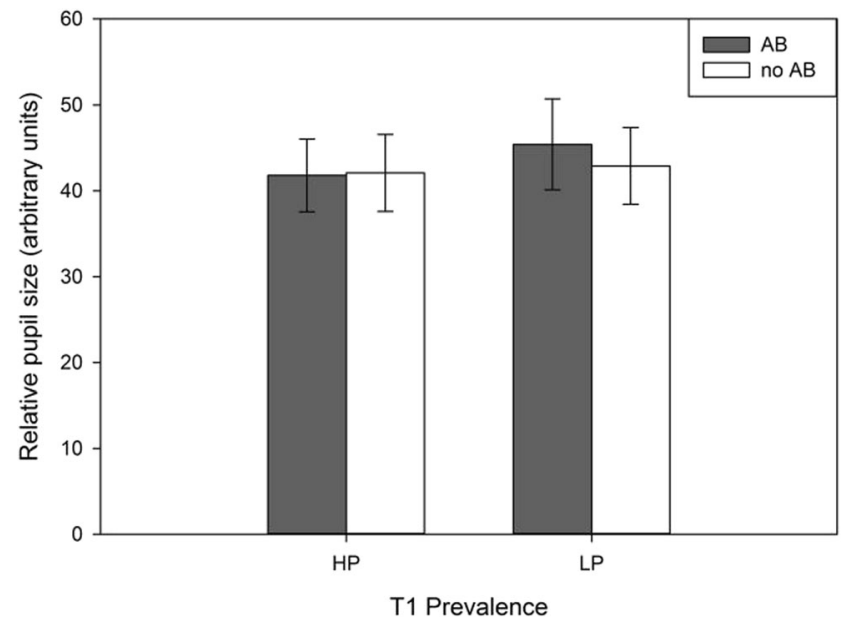

Fig. 5 Average baseline-corrected peak pupil diameter following highprevalence and low-prevalence $\mathrm{T} 1$ as a function of whether an attentional blink was observed for that trial. Error bars represent \pm 1 SEM

\section{Discussion}

Although we did not observe prevalence effects in T1 identification, which was likely due to our use of a paradigm that eliminates the more common selection (early search termination) errors ('Peltier \& Becker, 2016), Experiment 2 nonetheless revealed carryover prevalence effects in $\mathrm{T} 2$ identification. Replicating Experiment 1, we found that successful identification of a second target is impaired following the detection of a rare, relative to common, T1. Experiment 2 tested two predictions derived from the LC-NE hypothesis of the $\mathrm{AB}$ ('Nieuwenhuis et al., 2005): 1) Additional targets presented during the phasic LC response to T1 (within $100-150 \mathrm{~ms}$ of T1) should show enhanced detection rates, consistent with lag-1 sparing, and 2) Additional targets presented during the LC refractory period ( $200-400 \mathrm{~ms}$ after $\mathrm{T} 1)$ should show a negative relationship between LC involvement and detection rates, such that greater LC engagement yields worse performance. Our results confirmed the first prediction, replicating previous research showing lag-1 sparing (e.g., 'Visser et al., 1999). Although the LC-NE hypothesis predicts lag-1 sparing, greater engagement of the $\mathrm{LC}$ following rare $\mathrm{T} 1$ would predict enhanced lag-1 sparing following low-prevalence T1. Lag-1 detection rates were near ceiling in Experiment 2, however, possibly occluding any differential behavioral effects related 


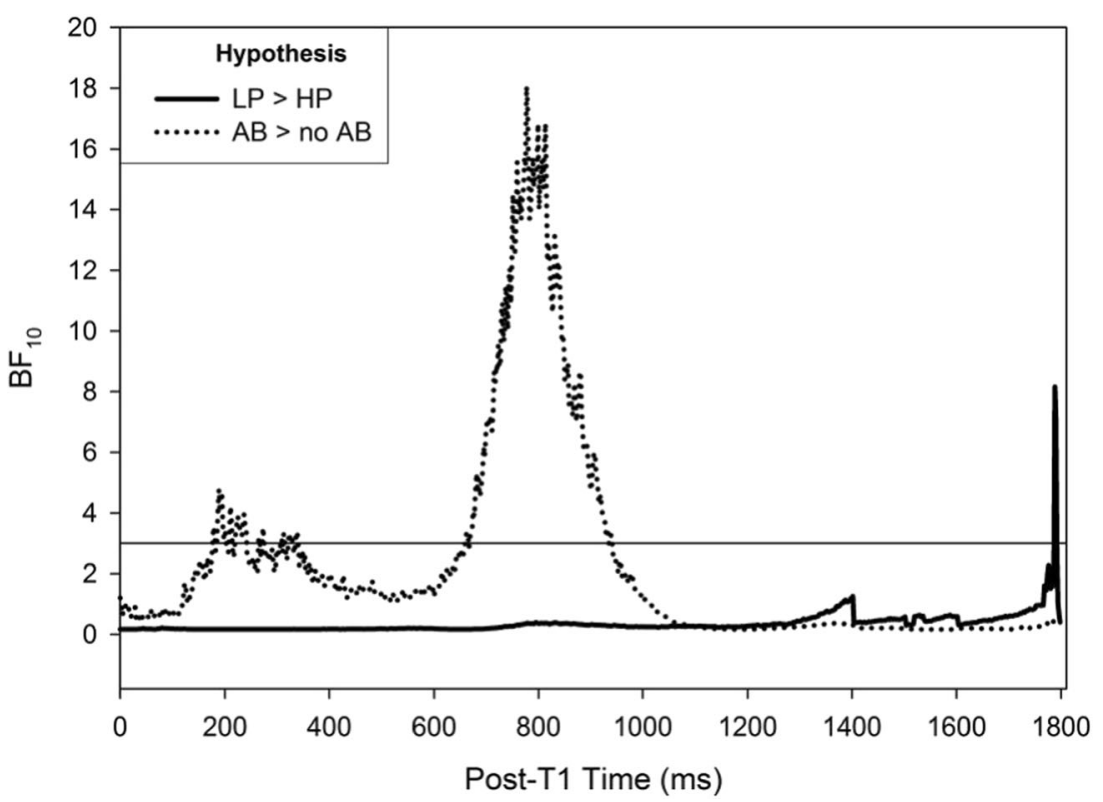

Fig. 6 Time-series Bayes factors $\left(\mathrm{BF}_{10}\right)$ evaluating the hypotheses that low-prevalence (LP) trials would yield larger pupils than high-prevalence (HP) trials (solid line) and attentional blink (AB) trials would yield larger

to T1 prevalence. Our results also confirmed the second hypothesis, and contribute new knowledge about the attentional costs and consequences of low-prevalence target detection. Specifically, we found that, even when LP targets were identified, they nonetheless resulted in greater attentional costs to subsequent processing, as reflected both behaviorally and via pupil diameter. Temporal analyses confirmed the time-course of these effects, with evidence for enhanced LC engagement pupils than no $\mathrm{AB}$ trials (dashed line) would. The horizontal reference line at $\mathrm{BF}_{10}=3$ indicates moderate evidence for the hypothesis (Jeffreys, 1961)

beginning $700-\mathrm{ms}$ post-T1 and ending by 900 -ms post-T1, consistent with the LC refractory period identified by 'Nieuwenhuis et al. (2005) and the temporal dynamics of phasic pupillary responses ('Joshi et al., 2016). Although we did not observe enlarged pupils for rare, relative to common, targets throughout the entire time series, these analyses were time-locked to T1 onset, which occurred in variable positions, potentially masking transient prevalence-mediated changes in

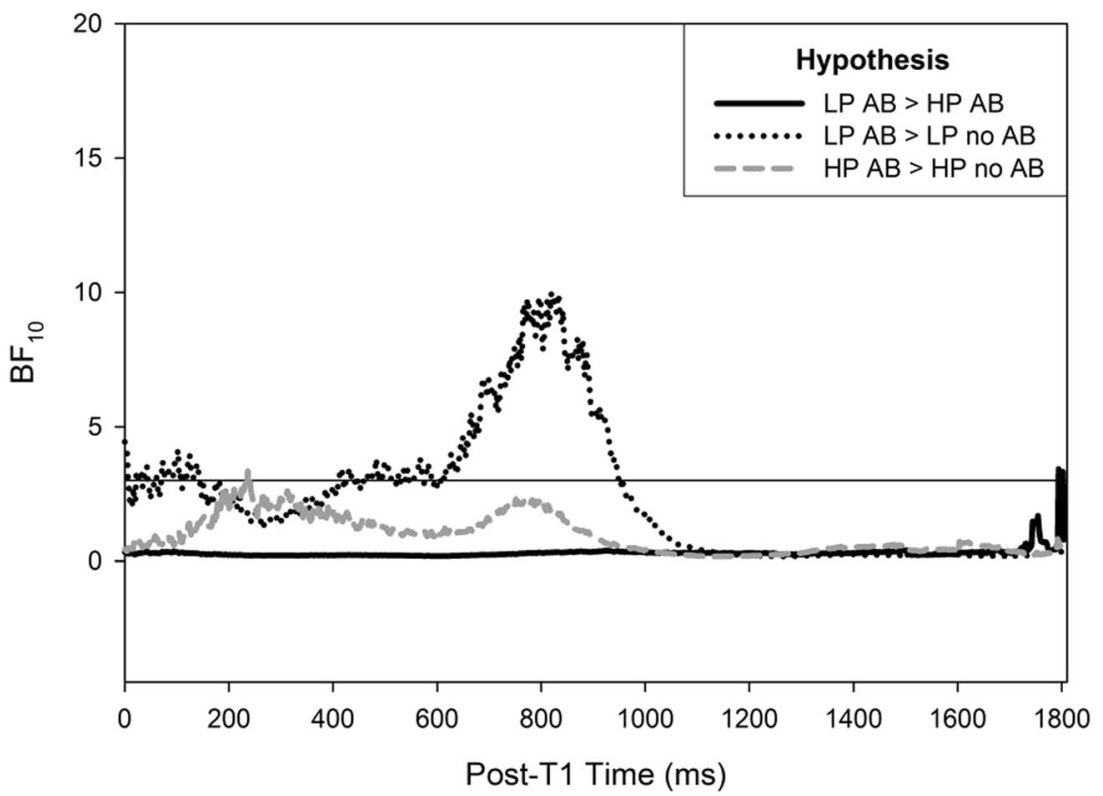

Fig. 7 Time-series Bayes factors $\left(\mathrm{BF}_{10}\right)$ evaluating the hypotheses that (1) low-prevalence (LP) attentional blink $(\mathrm{AB})$ trials would yield larger pupils than high-prevalence (HP) AB trials (solid line) would; (2) LP AB trials would yield larger pupils than LP no AB trials (dotted line) would; and (3) HP AB trials would yield larger pupils than HP no AB trials (dashed line) would 
attentional engagement (e.g., as when T1 occurred late in the stream). We discuss the theoretical impact of these findings in the General Discussion.

\section{General discussion}

In the present study, we examined the "downstream" consequences of the low-prevalence effect (LPE), which typically manifests as inflated miss rates to visual search targets that only rarely appear ('Wolfe et al., 2005). Although many studies have explored the causes of rare target misses (e.g., 'Fleck \& Mitroff, 2007; 'Wolfe \& Van Wert, 2010), we examined successful rare target detection, and its consequences for the availability of attentional resources for subsequent cognitive processing. Specifically, using a multiple-target RSVP paradigm, we eliminated the possibility of motor response errors (e.g., 'Fleck \& Mitroff, 2007) and relaxed quitting thresholds (e.g., 'Wolfe \& Van Wert, 2010), leaving only the possibility of conservative identification criteria. Although this eliminated a common source of LPE errors, rare targets (T1) were nevertheless detected less often than common targets in Experiment 1, consistent with research showing prevalence effects even when rare targets are directly viewed (Godwin et al., 2015a; 'Hout et al., 2015; 'Peltier \& Becker, 2016). Although Experiment 2 failed to replicate this effect, such that common and rare $\mathrm{T} 1$ were identified equally often, our primary interest was examining performance to the second target (T2) in the RSVP search stream, contingent upon successful T1 identification.

By focusing on $\mathrm{T} 2$ identification, our paradigm was closely aligned with research on the attentional blink $(\mathrm{AB})$, a phenomenon wherein observers fail to identify targets that occur in close temporal proximity to earlier targets ('Raymond et al., 1992). The AB is often theorized to reflect depletion of limited-capacity attentional resources by $\mathrm{T} 1$, leaving insufficient resources remaining to detect and identify T2 ('Martens $\&$ Wyble, 2010). Neurocomputational models of the AB relate this resource depletion to activity in the brain-stem locus coeruleus (LC), a neuromodulatory nucleus critically involved in regulating attention and cognition via norepinephrine (NE; 'Nieuwenhuis et al., 2005). When observers encounter motivationally relevant stimuli, the LC exhibits a phasic period of activity, transiently increasing the availability of NE. This phasic NE release is said to facilitate processing by increasing neural gain, temporarily making activated regions more sensitive to afferent input ('Berridge \& Waterhouse, 2003). Enhanced neural gain facilitates T1 detection, and can also explain lag-1 sparing (Niewenhuis et al., 2005), which occurs when $\mathrm{T} 2$ processing is spared if it occurs immediately after T1. More importantly, following phasic activity, the LC becomes inhibitory for a period approximately $200-400 \mathrm{~ms}$ postT1 ('Aston-Jones et al., 1994; 'Usher et al., 1999), which is closely related to the typical period of the AB ('Martens \& Wyble, 2010).

Nieuwenhuis et al. (2005) confirmed the presence of both the $\mathrm{AB}$ and lag-1 sparing in their computational model of LC function, and theorized that any stimulus property that differentially engages the LC should also differentially affect the $\mathrm{AB}$. We tested this hypothesis by manipulating stimulus frequency, a variable known to influence LC activity (AstonJones et al., 1997; 'Aston-Jones et al., 1994) and measuring the behavioral and pupillometric consequences of rare target detection. In Experiment 1, we confirmed the presence of an LPE in T1 detection, and showed that successful identification of rare $\mathrm{T} 1$ induces a stronger $\mathrm{AB}$, behaviorally consistent with the predictions of the LC-NE model. Experiment 2 further elucidated the impact of stimulus frequency by showing a more pronounced $\mathrm{AB}$ and effects of stimulus frequency on pupil size, a marker of LC activity ('Joshi et al., 2016). When observers encountered rare T1, they were less likely to identify an additional target presented within the LC refractory period, and this was marked by enlarged pupils. The time course of the effects documented in Figures 6 and 7 is similar to the period of autoinhibition that occurs following phasic bursts of LC activity ('Aghajanian et al., 1977; 'Egan et al., 1983; 'Washburn \& Moises, 1989), which causes pupils to enlarge, and suggests that the $\mathrm{AB}$ arises, at least in part, from the LC refractory period.

Most theories of the $\mathrm{AB}$ posit some form of limited attentional resource whose temporary depletion produces the characteristic "blink" in perception (e.g., 'Chun \& Potter, 1995; 'Di Lollo, Kawahara, Ghorashi, \& Enns, 2005; 'Jolicoeur, 1998, 1999; 'Shapiro, Raymond, \& Arnell, 1997), and many computational models have been put forth to explain the phenomenon (e.g., 'Bowman \& Wyble, 2007; 'Dehaene, Sergent, \& Changeux, 2003; 'Nieuwenhuis et al., 2005; 'Olivers \& Meeter, 2008; 'Shih, 2008; 'Simione et al., 2012). The LCNE model ('Nieuwenhuis et al., 2005), with which we see our results as consistent, is not unique in its ability to accommodate the dynamic facilitation (lag-1 sparing) and inhibition (AB) typically observed. Indeed, the boost and bounce theory (Olivers \& Meeter, 2008) has been proposed to accommodate both of these findings, as well as the necessity of distractors for producing the AB (e.g., 'Kawahara, Kumada, \& Di Lollo, 2006). Boost and bounce theory explains the characteristics of the $A B$ via interacting bottom-up and top-down attentional processes. When targets are encountered, bottom-up excitatory processes and top-down attentional enhancement give rise to target identification. These facilitation effects are not halted until a distractor engages bottom-up inhibitory processes, which themselves are inappropriately enhanced by remnant excitation from T1. In this way, the boost and bounce theory does not assume that limited-capacity mechanisms account for the $\mathrm{AB}$, but rather proposes that the effects arise due to attentional selection and gating mechanisms. Findings that have 
typically been taken as support for the boost and bounce theory, such as cuing effects (e.g., 'Nieuwenstein, 2006), have been explained within the context of the LC-NE model, and subsequent research on target-distractor interference ('Warren et al., 2009) has produced results inconsistent with boost and bounce. Our results, however, can be accommodated by both models of the AB: Whether infrequent $\mathrm{T} 1$ exacerbate the $\mathrm{LC}$ refractory period or produce greater post-T1 inhibition stemming from $\mathrm{T} 1$ excitation remains a question for future research.

Overall, our results provide clear evidence that the LPE manifests as more than just an inflated miss rate: When observers successfully detected rare $\mathrm{T} 1$, subsequent $\mathrm{T} 2$ detection was less accurate, relative to trials with common $\mathrm{T} 1$, suggesting that rare targets demand excess resources. This explanation has precedent within the literature on multiple-target search. For example, 'Adamo, Cain, and Mitroff (2017) explored individual differences in susceptibility to subsequent search misses (e.g., when a radiologist fails to spot a second target after detecting the first), and found that attentional depletion was a primary contributor to these miss rates. Similarly, eye-tracking research has revealed that, whereas common targets are located and identified quickly, rare targets take more time to identify, and are sometimes still "missed," despite direct fixation (e.g., Hout et al., 2015). In essence, the attentional processes consumed by rare target detection seem to make the human mind "less ready" to perceive another target, even when it is directly fixated. Although existing models of visual search (e.g., MDM; 'Wolfe \& Van Wert, 2010) nicely capture the causes of prevalence-linked miss rates (e.g., identification and quitting thresholds), the current results reveal that inflated miss rates are not the only consequence of low target prevalence. When observers successfully identify rare targets, their ability to process additional information is temporarily depleted.

Acknowledgements We thank Steve Walenchok and Steve Goldinger for providing feedback on an earlier version of the manuscript, and Ronen Hershman for sharing insights about CHAP and our pupillometry analyses. We are grateful to Qiraat Alsam, Torrance Thomas, Dynna Washington, Derek Hebert, Jonathan Dixon, and Amelia-Kate Johnson for their assistance with data collection.

Open practices statement The data and materials are available from the corresponding author. None of the experiments was preregistered.

Author contributions Both authors developed the study concept and contributed to the study design. J. D. Guevara Pinto oversaw data collection and performed and wrote about behavioral data analysis. M. H. Papesh analyzed the pupillometry data and wrote the manuscript. All authors have approved the final version for submission.

Funding Support provided by NIH/NICHD Grant No. R01 HD07580004.

\section{References}

Adamo, S. H., Cain, M. S., \& Mitroff, S. R. (2017). An individual differences approach to multiple-target visual search errors: How search errors relate to different characteristics of attention. Vision Research, 141, 258-265.

Aghajanian, G. K., Cedarbaum, J. M., \& Wang, R. Y. (1977). Evidence for norepinephrine-mediated collateral inhibition of locus coeruleus neurons. Brain Research, 136, 570-577.

Aston-Jones, G., \& Bloom, F. E. (1981). Norepinephrine-containing locus coeruleus neurons in behaving rats exhibit pronounced responses to non-noxious environmental stimuli. Journal of Neuroscience, 1, 887-900.

Aston-Jones, G., \& Cohen, J. D. (2005). Adaptive gain and the role of the locus coeruleus-norepinephrine system in optimal performance. The Journal of Comparative Neurology, 493, 99-110.

Aston-Jones, G., Rajkowski, J., \& Cohen, J. (1999). Role of locus coeruleus in attention and behavioral flexibility. Biological Psychiatry, 46(9), 1309-1320.

Aston-Jones, G., Rajkowski, J., \& Cohen, J. D. (2000). Locus coeruleus and regulation of behavioral flexibility and attention. Progress in Brain Research, 126, 165-182.

Aston-Jones, G., Rajkowski, J., \& Kubiak, P. (1997). Conditioned responses of monkey locus coeruleus neurons anticipate acquisition of discriminative behavior in a vigilance task. Neuroscience, 80(3), 697-715.

Aston-Jones, G., Rajkowski, J., Kubiak, P., \& Alexinsky, T. (1994). Locus coeruleus neurons in monkey are selectively activated by attended cues in a vigilance task. Journal of Neuroscience, 14, $4467-4480$.

Beaudet, A., \& Descarries, L. (1978). The monoamine innervation of rat cerebral cortex: Synaptic and nonsynaptic axon terminals. Neuroscience, 3(10), 851-860.

Berridge, C. W., \& Waterhouse, B. D. (2003). The locus coeruleusnoradrenergic system: Modulation of behavioral state and statedependent cognitive processes. Brain Research Reviews, 42(1), 33-84.

Bickler, P. E., \& Hansen, B. M. (1996). Alpha 2-adrenergic agonists reduce glutamate release and glutamate receptor-mediated calcium changes in hippocampal slices during hypoxia. Neuropharmacology, 35, 679-87.

Bowman, H., \& Wyble, B. (2007). The simultaneous type, serial token model of temporal attention and working memory. Psychological Review, 114(1), 38-70.

Chun, M. M., \& Potter, M. C. (1995). A two-stage model for multiple target detection in rapid serial visual presentation. Journal of Experimental Psychology: Human Perception and Performance, 21(1), 109-127.

Clayton, E. C., Rajkowski, J., Cohen, J. D., \& Aston-Jones, G. (2004). Phasic activation of monkey locus coeruleus neurons in a forcedchoice task. Journal of Neuroscience, 24, 9914-9920.

Clewett, D., Huang, R., Velasco, R., Lee, T. H., \& Mather, M. (2018). Locus coeruleus activity strengthens prioritized memories under arousal. Journal of Neuroscience, 2097-3017. doi:https://doi.org/ 10.1523/JNEUROSCI.2097-17.2017

Crebolder, J. M., Jolicœur, P., \& McIlwaine, J. D. (2002). Loci of signal probability effects and of the attentional blink bottleneck. Journal of Experimental Psychology: Human Perception and Performance, 28(3), 695-716.

Dehaene, S., Sergent, C., \& Changeux, J. P. (2003). A neuronal network model linking subjective reports and objective physiological data during conscious perception. Proceedings of the National Academy of Sciences, 100(14), 8520-8525. 
Descarries, L., Watkins, K. C., \& Lapierre, Y. (1977) Noradrenergic axon terminals in the cerebral cortex of rat: III. Topometric ultrastructural analysis. Brain Research, 133, 197-222.

Di Lollo, V., Kawahara, J. I., Ghorashi, S. S., \& Enns, J. T. (2005). The attentional blink: Resource depletion or temporary loss of control? Psychological Research, 69(3), 191-200.

Egan, T. M., Henderson, G., North, R. A., \& Williams, J. T. (1983). Noradrenaline-mediated synaptic inhibition in rat locus coeruleus neurons. Journal of Physiology, 345, 477-488.

Egli, R. E., Kash, T. L., Choo, K., Savchenko, V., Matthews, R. T., Blakely, R. D., \& Winder, D. G. (2005). Norepinephrine modulates glutamatergic transmission in the bed nucleus of the stria terminalis. Neuropsychopharmacology, 30(4), 657-668.

Evans, K. K., Birdwell, R. L., \& Wolfe, J. M. (2013). If you don't find it often, you often don't find it: Why some cancers are missed in breast cancer screening. PLOS ONE, 8(5), e64366. doi:https://doi.org/10. 1371/journal.pone.0064366

Fleck, M., \& Mitroff, S. R. (2007). Rare targets are rarely missed in correctable search. Psychological Science, 18, 943-947.

Fleck, M. S., Samei, E., \& Mitroff, S. R. (2010). Generalized "satisfaction of search": Adverse influences on dual-target search accuracy. Journal of Experimental Psychology: Applied, 16, 60-71. doi: https://doi.org/10.1037/a0018629

Foote, S. L., Aston-Jones, G., \& Bloom, F. E. (1980). Impulse activity of locus coeruleus neurons in awake rats and monkeys is a function of sensory stimulation and arousal. Proceedings of the National Academy of Sciences, 77, 3033-3037.

Foote, S. L., Freedman, R., \& Oliver, A. P. (1975) Effects of putative neurotransmitters on neuronal activity in monkey auditory cortex. Brain Research, 86 (2), 229-242.

Freedman, R., Hoffer, B. J., Woodward, D. J., \& Puro, D. (1977) Interaction of norepinephrine with cerebellar activity evoked by mossy and climbing fibers. Experimental Neurology, 55(1), 269288

Gilzenrat, M. S., Holmes, B. D., Rajkowski, J., Aston-Jones, G., \& Cohen, J. D. (2002). Simplified dynamics in a model of noradrenergic modulation of cognitive performance. Neural Networks, 15, 647-663.

Gilzenrat, M. S., Nieuwenhuis, S., Jepma, M., \& Cohen, J. D. (2010). Pupil diameter tracks changes in control state predicted by the adaptive gain theory of locus coeruleus function. Cognitive, Affective, \& Behavioral Neuroscience, 10, 252-269.

Godwin, H. J., Menneer, T., Riggs, C. A., Cave, K. R., \& Donnelly, N. (2015a). Perceptual failures in the selection and identification of low-prevalence targets in relative prevalence visual search. Attention, Perception, and Psychophysics, 77, 150-159. doi: https://doi.org/10.3758/s13414-014-0762-8

Godwin, H. J., Menneer, T., Riggs, C. A., Cave, K. R., Thaibsyah, M., \& Donnelly, N. (2015b). The effects of increasing target prevalence on information processing during visual search. Psychonomic Bulletin \& Review, 22, 469-475.

Grant, S. J., Aston-Jones, G., \& Redmond, D. E. J. (1988). Responses of primate locus coeruleus neurons to simple and complex sensory stimuli. Brain Research Bulletin, 21, 401-410.

Hershman, R., Henik, A., \& Cohen, N. (2019). CHAP: Open-source software for processing and analyzing pupillometry data. Behavior Research Methods, 1-16.

Horowitz, T. S. (2017). Prevalence in visual search: From the clinic to the lab and back again. Japanese Psychological Research, 59(2), 65108. doi:https://doi.org/10.1111/jpr.12153

Hout, M. C., Walenchok, S. C., Goldinger, S. D., \& Wolfe, J. M. (2015). Failures of perception in the low-prevalence effect: Evidence from active and passive visual search. Journal of Experimental Psychology: Human Perception and Performance, 41, 977-994. doi:https://doi.org/10.1037/xhp0000053
JASP Team. (2018). JASP (Version 0.9)[Computer software]. Retrieved from https://jasp-stats.org/2018/06/20/introducing-jasp-0-9/

Jolicoeur, P. (1998). Modulation of the attentional blink by on-line response selection: Evidence from speeded and unspeeded Task 1 decisions. Memory \& Cognition, 26(5), 1014-1032.

Jolicoeur, P. (1999). Concurrent response-selection demands modulate the attentional blink. Journal of Experimental Psychology: Human Perception and Performance, 25(4), 1097-1113.

Joshi, S., Li, Y., Kalwani, R. M., \& Gold, J. I. (2016). Relationships between pupil diameter and neuronal activity in the locus coeruleus, colliculi, and cingulate cortex. Neuron, 89, 221-234.

Kawahara, J.-I., Kumada, T., \& Di Lollo, V. (2006). The attentional blink is governed by a temporary loss of control. Psychonomic Bulletin \& Review, 13, 886-890.

Martens, S., \& Wyble, B. (2010). The attentional blink: Past, present, and future of a blind spot of perceptual awareness. Neuroscience \& Biobehavioral Reviews, 34(6), 947-957. doi:https://doi.org/10. 1016/j.neubiorev.2009.12.005

Morrison, J. H., \& Foote, S. L. (1986). Noradrenergic and serotoninergic innervation of cortical, thalamic, and tectal visual structures in Old and New World monkeys. Journal of Comparative Neurology, 243(1), 117-138.

Nieuwenhuis, S., Gilzenrat, M. S., Holmes, B. D., \& Cohen, J. D. (2005) The role of the locus coeruleus in mediating the attentional blink: A neurocomputational theory. Journal of Experimental Psychology: General, 134(3), 291-307. doi:https://doi.org/10.1037/0096-3445. 134.3.291

Nieuwenstein, M. R. (2006). Top-down controlled, delayed selection in the attentional blink. Journal of Experimental Psychology: Human Perception and Performance, 32(4), 973-985.

O’Donnell, J., Zeppenfeld, D., McConnell, E., Pena, S., \& Nedergaard, M. (2012). Norepinephrine: A neuromodulator that boosts the function of multiple cell types to optimize CNS performance. Neurochemical Research, 37(11), 2496-2512.

Olivers, C. N., \& Meeter, M. (2008). A boost and bounce theory of temporal attention. Psychological Review, 115(4), 836-863. doi: https://doi.org/10.1037/a0013395

Papesh, M. H., Heisick, L. L., \& Warner, K. M. (2018). The lowprevalence effect in unfamiliar face-matching: The roles of feedback and criterion shifting. Journal of Experimental Psychology: Applied, 24(3), 416-430.

Peltier, C., \& Becker, M. W. (2016). Decision processes in visual search as a function of target prevalence. Journal of Experimental Psychology: Human Perception and Performance, 42, 1466-1476. doi:https://doi.org/10.1037/xhp0000248

Polack, P.-O., Friedman, J., \& Golshani, P. (2013) Cellular mechanisms of brain state- dependent gain modulation in visual cortex. Nature Neuroscience, 16(9), 1331-1339.

Potter, M. C., Staub, A., \& O'Connor, D. H. (2002). The time course of competition for attention: Attention is initially labile. Journal of Experimental Psychology: Human Perception \& Performance, 28, 1149-1162. doi:https://doi.org/10.1037//0096-1523.28.5.1149

Psychology Software Tools, Inc. E-Prime 2.0 [Computer software]. (2012). Sharpsburg, PA: Author. Retrieved from http://www. pstnet.com

Ramos, B. P., \& Arnsten, A. F. T. (2007) Adrenergic pharmacology and cognition: Focus on the prefrontal cortex. Pharmacology and Therapeutics, 113(3), 523-536.

Rasmussen, K., Morilak, D. A., \& Jacobs, B. L. (1986). Single unit activity of locus coeruleus neurons in the freely moving cat: I. During naturalistic behaviors and in response to simple and complex stimuli. Brain Research, 371, 324-334.

Raymond, J. E., Shapiro, K. L., \& Arnell, K. M. (1992). Temporary suppression of visual processing in an RSVP task: An attentionalblink? Journal of Experimental Psychology: Human Perception and 
Performance, 18(3), 849-860. doi:https://doi.org/10.1037/00961523.18.3.849

Rich, A. N., Kunar, M. A., Van Wert, M. J., Hidalgo-Sotelo, B., Horowitz, T. S., \& Wolfe, J. M. (2008). Why do we miss rare targets? Exploring the boundaries of the low prevalence effect. Journal of Vision, 8, 1-17. doi:https://doi.org/10.1167/8.15.15

Sara, S. J., \& Segal, M. (1991). Plasticity of sensory responses of locus coeruleus neurons in the behaving rat: Implications for cognition. Progress in Brain Research, 88, 571-585.

Segal, M., \& Bloom, F. E. (1976) The action of norepinephrine in the rat hippocampus: IV. The effects of locus coeruleus stimulation on evoked hippocampal unit activity. Brain Research, 107(3), 513525 .

Shapiro, K. L., Raymond, J. E., \& Arnell, K. M. (1994). Attention to visual pattern information produces the attentional blink in rapid serial visual presentation. Journal of Experimental Psychology: Human Perception and Performance, 20(2), 357-371.

Shapiro, K. L., Raymond, J. E., \& Arnell, K. M. (1997). The attentional blink. Trends in Cognitive Sciences, 1(8), 291-296.

Shih, S.-I. (2008). The attention cascade model and the attentional blink. Cognitive Psychology, 56, 210-236.

Simione, L., Raffone, A., Wolters, G., Salmas, P., Nakatani, C., Belardinelli, M. O., \& van Leeuwen, C. (2012). ViSA: A neurodynamic model for visuo-spatial working memory, attentional blink, and conscious access. Psychological Review, 119(4), 745769. doi:https://doi.org/10.1037/a0030050

Usher, M., Cohen, J. D., Servan-Schreiber, D., Rajkowski, J., \& AstonJones, G. (1999). The role of locus coeruleus in the regulation of cognitive performance. Science, 283, 549-554.

Visser, T. A. W., Bischof, W. F., \& Di Lollo, V. (1999). Attentional switching in spatial and nonspatial domains: Evidence from the attentional blink. Psychological Bulletin, 125, 458-469. doi: https://doi.org/10.1037/0033-2909.125.4.458

Warren, C. M., Breuer, A. T., Kantner, J., Fiset, D., Blais, C., \& Masson, M. E. (2009). Target-distractor interference in the attentional blink implicates the locus coeruleus-norepinephrine system. Psychonomic Bulletin \& Review, 16(6), 1106-1111. doi:https://doi. org/10.3758/PBR.16.6.1106

Washburn, M., \& Moises, H. C. (1989). Electrophysiological correlates of presynaptic alpha 2-receptor-mediated inhibition of norepinephrine release at locus coeruleus synapses in dentate gyrus. Journal of Neuroscience, 9, 2131-2140.

Waterhouse, B. D., \& Woodward, D. J. (1980) Interaction of norepinephrine with cerebrocortical activity evoked by stimulation of somatosensory afferent pathways in the rat. Experimental Neurology, 67(1), $11-34$.

Wierda, S. M., van Rijn, H., Taatgen, N. A., \& Martens, S. (2012). Pupil dilation deconvolution reveals the dynamics of attention at high temporal resolution. Proceedings of the National Academy of Sciences, 109(22), 8456-8460. doi:https://doi.org/10.1073/pnas. 1201858109

Wolfe, J. M., Brunelli, D. N., Rubinstein, J., \& Horowitz, T. S. (2013). Prevalence effects in newly trained airport checkpoint screeners: Trained observers miss rare targets, too. Journal of Vision, 13(3), 1-9. doi:https://doi.org/10.1167/13.3.33

Wolfe, J. M., Horowitz, T. S., \& Kenner, N., M. (2005). Rare items often missed in visual searches. Nature, 435, 439-440. doi:https://doi.org/ $10.1038 / 435439 a$

Wolfe, J. M., Horowitz, T. S., Van Wert, M. J., Kenner, N. M., Place, S. S., \& Kibbi, N. (2007). Low target prevalence is a stubborn source of errors in visual search tasks. Journal of Experimental Psychology: General, 136, 623-638. doi:https://doi.org/10.1037/0096-3445.136. 4.623

Wolfe, J. M., \& Van Wert, M. J. (2010). Varying target prevalence reveals two dissociable decision criteria in visual search. Current Biology, 20,121-124. doi:https://doi.org/10.1016/j.cub.2009.11.066

Zylberberg, A., Oliva, M., \& Sigman, M. (2012). Pupil dilation: A fingerprint of temporal selection during the attentional blink." Frontiers in Psychology, 3, 316, 1-6. doi:https://doi.org/10.3389/ fpsyg.2012.00316

Publisher's note Springer Nature remains neutral with regard to jurisdictional claims in published maps and institutional affiliations. 